\title{
Universal temperature corrections to the free energy for the gravitational field
}

\author{
G.E. Volovik \\ Low Temperature Laboratory, Helsinki University of Technology \\ Box 2200, FIN-02015 HUT, Finland \\ and \\ L.D. Landau Institute for Theoretical Physics, \\ 117334 Moscow, Russia \\ and \\ A. Zelnikov \\ Theoretical Physics Institute, University of Alberta \\ Edmonton, Alberta, Canada T6G 2J1 \\ and \\ P.N. Lebedev Physics Institute \\ Leninskii prospect 53, Moscow 119991 Russia
}

November 5, 2018

\begin{abstract}
The temperature correction to the free energy of the gravitational field is considered which does not depend on the Planck energy physics. The leading correction may be interpreted in terms of the temperature dependent effective gravitational constant $G_{\text {eff }}$. The temperature correction to $G_{\text {eff }}^{-1}$ appears to be valid for all temperatures $T \ll E_{\text {Planck }}$. It is universal since it is determined only by the number of fermionic and bosonic fields with masses $m \ll T$, does not contain the Planck energy scale $E_{\text {Planck }}$ which determines the gravitational constant at $T=0$, and does not depend on whether or not the gravitational field
\end{abstract}


obeys the Einstein equations. That is why this universal modification of the free energy for gravitational field can be used to study thermodynamics of quantum systems in condensed matter (such as quantum liquids superfluid ${ }^{3} \mathrm{He}$ and ${ }^{4} \mathrm{He}$ ), where the effective gravity emerging for fermionic and/or bosonic quasiparticles in the low-energy corner is quite different from the Einstein gravity.

\section{Introduction}

The corrections to the Einstein action for the gravitational field typically depend on the underlying physics of the gravity. There are many ways to produce the gravity: the gravity, of course, can be the fundamental field; but it also can be an effective field induced by quantum fluctuations of massive bosonic or fermionic fields according to the Sakharov scenario $[1,2,3]$; it can gradually emerge in the low-energy corner of the fermionic system with massless topologically protected fermions [4]; the metric/vierbein fields can emerge also as a result of the symmetry breaking as a vacuum expectation value of the bilinear combimations of spinor fields [5]; etc. In most cases the corrections to the Einstein action are non-universal since they depend on the trans-Planckian physics. However, there are special cases when the corrections are completely determined by the infrared physics. These corrections are universal since they do not depend on the details of the underlying physics, and are the same irrespective of whether the gravity is fundamental or effective. Here we consider such universal corrections to the free energy for the gravitational field which come from the infrared fermionic and/or bosonic fields whose masses are small compared to the temperature $T$.

The same universal terms in the free energy for the effective gravity arise in the condensed matter systems, in spite of the fact that the main action which governs the effective metric does not resemble the Einstein acton even remotely. We discuss such subdominating terms in the bosonic superfluid ${ }^{4} \mathrm{He}$ and fermionic superfluid ${ }^{3} \mathrm{He}$, where the effective metric field emerges for the bosonic and fermionic quasiparticles respectively. Since the temperature in these liquids is not very small compared to the corresponding 'Planck'

scale $E_{\text {Planck }}$ (which is typically the Debye temperature or the temperature of superfluid transition $T_{c}$ ), the effect of these terms is accesible for experiments. This allows us to experimentally verify many issues of the interplay of the 
gravity and thermodynamics.

\section{Contribution of massless fermions}

\section{$2.1 \quad$ Relativistic theory}

Let us start with the modification of the free energy for gravity which comes from the massless fermions. Here we are interested in the contribution which comes from the thermal relativistic fermions in the background of the curved space. The free energy of the relativistic gas of fermions in the stationary gravitational field has the correction containing the Ricci curvature $\mathcal{R}$ of the gravitational field. If the gas is in the global equilibrium with temperature $T_{0}$ at infinity one obtains in the high-temperature limit $T^{2} \gg \hbar^{2} \mathcal{R}$ (but $\left.T \ll E_{\text {Planck }}\right)$ the following free energy $[6,8,9]$ :

$$
F=F_{0}-\frac{7 \pi^{2} N_{F}}{360 \hbar^{3}} \int d^{3} x \sqrt{-g} T^{4}+\frac{N_{F}}{288 \hbar} \int d^{3} x \sqrt{-g} T^{2}\left[\mathcal{R}+6 w^{2}\right] .
$$

Here $F_{0}$ is the temperature independent bulk part of the free energy of a static gravitational field ${ }^{1}$

$$
F_{0}=-\frac{1}{16 \pi G} \int d^{3} x \sqrt{-g} \mathcal{R}
$$

The $T^{4}$-term in Eq.(1), $F_{4}$, is the thermal free energy of massless chiral fermions, where $N_{F}$ is the number of different fermionic species (alternatively $N_{F}=2 N_{D}$ for $N_{D}$ Dirac fermions with masses $\left.m \ll T\right)$. The temperature $T$ is a local (red-shifted) temperature obeying the Tolman's law

$$
T(\mathbf{r})=\frac{T_{0}}{\sqrt{\left|g_{00}(\mathbf{r})\right|}},
$$

and $T_{0}=$ Const.

\footnotetext{
${ }^{1}$ The main bulk contribution to $F_{0}$ comes from the space integral of the Einstein lagrangian taken with the minus sign. This follows from the relation of the free energy to the Euclidean action $F=T I_{\text {Eucl }}[10]$ and the definition of the Euclidean Einstein action $-\frac{1}{16 \pi G} \int d^{4} x \sqrt{g} \mathcal{R}$ with the overall minus sign compare to the Einstein action in Minkowskian signature [11].
} 
The mixed thermal-gravitational $T^{2}$-term $F_{2}$ in Eq.(1) is the subject of our discussion. Here $w^{2}=w^{\mu} w_{\mu}$, where $w_{\mu}=\frac{1}{2} \partial_{\mu} \ln \left|g_{00}(\mathbf{r})\right|$ is the 4acceleration. The temperature dependent part of the free energy, $F_{2}+F_{4}$, is invariant under stationary conformal transformations [6]. This is because the thermodynamics is determined by the (quasi)particle spectrum, and in the static spacetimes the energy spectrum $E^{2}=-p_{i} p_{k} g^{i k} / g^{00}$ does not depend on the conformal factor. The $6 w^{2}$ term in Eq.(1) provides the conformal invariance of the $F_{2}$-term.

The total free energy can be represented in terms of the effective gravitational 'constant' $G_{\text {eff }}$

$$
F=-\frac{7 \pi^{2} N_{F}}{360 \hbar^{3}} \int d^{3} x \sqrt{-g}\left(T^{4}-\frac{15}{14 \pi^{2}} T^{2} w^{2}\right)-\frac{1}{16 \pi} \int d^{3} x \frac{1}{G_{\text {eff }}} \sqrt{-g} \mathcal{R},
$$

which is renormalized due to the gravitational polarization of matter and becomes coordinate dependent due to Tolman's law (3):

$$
G_{\text {eff }}^{-1}(\mathbf{r})-G^{-1}(T=0)=-\frac{\pi N_{F}}{18 \hbar} T^{2}(\mathbf{r}) .
$$

Alternatively one could define $G_{\text {eff }}$ as prefactor in front of the curvature terms in the equations obtained by variation of the free energy $\delta F / \delta g^{\mu \nu}(\mathbf{r})=0$. However, this dynamical definition of $G_{\text {eff }}$ does not make much sense for our problems since the effective metric felt by quasiparticles in condensed matter does not satisfy the Einstein equations anyway. Thermodynamcal quantities are more robust in this case. That is why here and further we determine $G_{\text {eff }}^{-1}$ as the prefactor in front of the curvature $\mathcal{R}$ in the free energy.

It should be mentioned that Eq.(4) is valid for the static (or stationary) case only, since we discuss the system in a global equilibrium. For the same reason the $\mathcal{R} T^{2}$ correction, in spite of its formally generally-covariant appearance, in fact is not generally-covariant, since in a global equilibrium the reference frame is fixed. Just for the same reason the free energy for the gauge field violates the Lorentz invariance as was discussed for the static electromagnetic field in Ref. [7].

Variation of the free energy over the stationary metric $g^{\mu \nu}(\mathbf{r})$ gives the equations for the stationary metric fields in a global equilibrium, i.e. at fixed $T_{0}$. Because of the temperature corrections to the gravitational 'constant', $G_{\text {eff }}$ itself depends on the metric element $g_{00}$. As a result the obtained equations do not coincide with the classical Einstein equations $G_{\mu \nu}=8 \pi G_{\mathrm{eff}} T_{\mu \nu}^{\mathrm{M}}$, 
where $G_{\mu \nu}$ is the Einstein tensor and $T_{\mu \nu}^{\mathrm{M}}$ is the energy-momentum tensor of matter. Instead one has

$$
G_{\mu \nu}=8 \pi G\left(T_{\mu \nu}^{\mathrm{M}}+T_{\mu \nu}^{\mathrm{M}+\mathrm{G}}\right)
$$

where $T_{\mu \nu}^{\mathrm{M}+\mathrm{G}}$ is non-covariant mixed thermal-gravitational term which depends both on the temperatute $T_{0}$ and on derivatives of the metric tensor. This means that the gravity in a medium is different from the gravity in the vacuum: the matter (here the relativistic massless fermions at given $T_{0}$ ) is not only the source of gravity, but it also modifies the gravitational interactions which depend on the reference frame in which the global equilibrium is achieved.

For us it is important that the mixed thermal-gravitational term $F_{2}$ in the free energy, and thus the temperature correction to the effective gravitational constant in Eq.(5) are the properties of the low-energy physics of the massless fields in the classical gravitational background: the $T^{2}$ correction is universal and does not depend on the physics at Planck scale. It is determined only by the number and type of massless fields which are present in the Universe at given temperature, and these fields may have nothing to do with the underlying quantum fields whose vacuum fluctuations contribute to the gravitational constant $G$ at $T=0$ in induced gravity. This means that the temperature correction does not know whether the gravity is fundamental or effective.

The effect of the polarization of matter in the gravitational field is extremely small in normal conditions, since the temperature is extremely small compared to the Planck energy scale. The renormalization may become important only when $g_{00} \rightarrow$ 0, i.e. when the system is close to the threshold of formation of the horizon or ergoregion. But in condensed matter the temperatures are typically not very low compared with the analogs of the Planck energy - the Debye temperature $T_{\text {Debye }} \sim 10 \mathrm{~K}$ in superfluid ${ }^{4} \mathrm{He}$; the superfluid transition temperature $T_{c} \sim 1 \mathrm{mK}$ in ${ }^{3} \mathrm{He}-\mathrm{A}$; and the transition temperature $T_{c} \sim 10-100 \mathrm{~K}$ in superconductors. That is why this effect can be pronounced in the effective gravity arising in condensed matter. Moreover, when the thermodynamics or kinetic properties of a condensed matter system are measured, such as the specific heat, entropy or heat conductivity, the $T=0$ contribution drops out and the temperature dependent terms become dominating. In the high temperature regime $T^{2} \gg \hbar^{2} \mathcal{R}$ the $T^{4}$ 
term is dominating in thermodynamics. However, the curvature simulated by textures in quantum liquids can be made so strong that the subdominating $T^{2} \mathcal{R}$ term under discussion becomes comparable to the $T^{4}$ term, and in principle even the opposite regime $T^{2} \ll \hbar^{2} \mathcal{R}$ can be reached which is called the texture-dominating regime in condensed matter.

Here we consider the analogs of the $T^{2} \mathcal{R}$ term on the example of two condensed matter systems.

\subsection{Chiral fermions in ${ }^{3} \mathrm{He}-\mathrm{A}$}

Let us start with ${ }^{3} \mathrm{He}-\mathrm{A}$ where the effective metric felt by chiral fermionic quasiparticles living in the vicinity of Fermi points (i.e. in the low-energy corner) is [4]

$$
g_{i j}=\frac{1}{c_{\|}^{2}} \hat{l}^{i} \hat{l}^{j}+\frac{1}{c_{\perp}^{2}}\left(\delta^{i j}-\hat{l}^{i} \hat{l}^{j}\right), g_{00}=-1, \sqrt{-g}=\frac{1}{c_{\|} c_{\perp}^{2}} .
$$

Here $\hat{\mathbf{l}}$ is the unit vector showing the axis of the uniaxial orbital anisotropy of the superfluid vacuum; the longitudinal and transverse 'speeds of light' are

$$
c_{\|}=\frac{p_{F}}{m^{*}} \quad, \quad c_{\perp}=\frac{\Delta_{0}}{p_{F}} \ll c_{\|},
$$

where $p_{F}$ and $p_{F} / m^{*}$ are Fermi momentum and Fermi velocity correspondingly, and $\Delta_{0}$ is the gap amplitude. We omitted the non-static elements $g_{0 i}$ produced by the superfluid velocity field, which will be discussed later for the Bose superfluids, and consider the contribution of only the soft Goldstone degrees of freedom related to the deformation of the unit vector $\hat{\mathbf{l}}$ (called textures in condensde matter). That is why the 'speeds of light' are considered as constants in space, as well as $g_{00}$, which may be chosen as $g_{00}=-1$. This corresponds to the ultrastatic spacetime in general relativity.

Omitting the total derivatives one can calculate the integral of the Ricci scalar of the metric in Eq.(7)

$$
\int d^{3} x \sqrt{-g} \mathcal{R}=-\frac{1}{2} \int d^{3} x c_{\|} c_{\perp}^{2}\left(\frac{1}{c_{\perp}^{2}}-\frac{1}{c_{\|}^{2}}\right)^{2}\left((\hat{\mathbf{l}} \cdot(\nabla \times \hat{\mathbf{l}}))^{2}+\right.\text { surface terms. }
$$


Let us consider how the term like that enters the free energy of ${ }^{3} \mathrm{He}-\mathrm{A}$. The gradient expansion of the free energy in terms of the gradients of $\hat{\mathbf{l}}$-field has been elaborated by Cross in the limit $c_{\perp} \ll c_{\|}[12]$. At that time it was not known that the effective gravity for quasiparticles emerges at low energy. But now when we look at the gradient energy derived by Cross we find that it does contain the twist term which quadratically depends on $T$ [4]:

$$
F_{2}=-\frac{1}{288 \hbar} \int d^{3} x \frac{c_{\|}}{c_{\perp}^{2}} T^{2}(\hat{\mathbf{l}} \cdot(\nabla \times \hat{\mathbf{l}}))^{2} \equiv \frac{1}{144 \hbar} \int d^{3} x \sqrt{-g} T^{2} \mathcal{R}
$$

Here $T=T_{0}$ since $g_{00}=-1$. The thermal part of the free energy does not contain microscopic (trans-Planckian) parameters, and is completely determined by the Planck constant $\hbar$ and by the parameters of the spectrum of fermionic quasiparticles in the low-energy limit when the spectrum becomes 'relativistic': $E^{2}=g^{i k} p_{i} p_{k}=c_{\|}^{2}(\mathbf{p} \cdot \hat{\mathbf{l}})^{2}+c_{\perp}^{2}(\mathbf{p} \times \hat{\mathbf{l}})^{2}$ (here the momentum $\mathbf{p}$ of quasiparticles is counted from the Fermi point). This term exactly coincides with the temperature correction in Eq.(1) in the limit $c_{\perp} \ll c_{\|}$, since the number of the massless chiral fermions living in the low-energy corner of ${ }^{3} \mathrm{He}-\mathrm{A}$ is $N_{F}=2$. This demonstrates the universality of the $T^{2} \mathcal{R}$-term, i.e. its independence of the trans-Planckian physics, while the term $F_{0}$ is essentially determined by the trans-Planckian physics [4].

We discussed the contribution to the effective action which comes from massless fermions. In ${ }^{3} \mathrm{He}-\mathrm{A}$ there are also analogs of massless photons and gauge bosons, moreover in the logarithmic approximation the effective electrodynamics obeys the same metric $g_{\mu \nu}$ as fermionic quasiparticles, and thus one could expect the similar contribution of the gauge bosons to $G_{\text {eff }}^{-1}$. However, this contribution has not been found. The reason for that is that in the effective electrodynamics emerging in ${ }^{3} \mathrm{He}-\mathrm{A}$ the non-renormalizable non-logarithmic terms are comparable with the covariant terms containing the logarithmically divergent running coupling [4]. As result the 'speed of light' for photons differs from the 'speed of light' for chiral fermions. While $c_{\|}$(gauge bosons) $=c_{\|}$(chiral fermions), the transverse speed of light is essentially bigger: $c_{\perp}$ (gauge bosons) $\gg c_{\perp}$ (chiral fermions). Since according to Eq.(10) $c_{\perp}$ is in the denominator, the contribution of the gauge bosons to the $T^{2} \mathcal{R}$ term is negligible.

The contribution of the scalar fields with their own (acoustic) metric will be discussed further in the paper. 


\section{Contribution of scalar fields}

\subsection{Bosonic field}

The origin of the effective gravity in superfluid ${ }^{4} \mathrm{He}$ is essentially different from that in ${ }^{3} \mathrm{He}-\mathrm{A}$. The superfluid ${ }^{3} \mathrm{He}-\mathrm{A}$ belongs to the same universality class of the fermionic systems as the quantum vacuum of the Standard Model, and one can expect that these systems have a common origin of the emerging gravity represented by the collective modes related to the deformation of Fermi points. The superfluid ${ }^{4} \mathrm{He}$ is the Bose-liquid where bosonic quasiparticles - phonons - play the role of a scalar field, propagating in the background of the effective metric $g_{\mu \nu}$ provided by the moving superfluid condensate (superfluid quantum vacuum). The gravity field $g_{\mu \nu}$ experienced by phonons is simulated mainly by the superfluid velocity field $\mathbf{v}_{\mathbf{s}}$ (velocity of the superfluid quantum vacuum). The dynamics of the velocity field is determined by the hydrodynamic equations instead of the Einstein equations. Nevertheless, the universal $T^{2} \mathcal{R}$ term must enter the effective action for $g_{\mu \nu}$, and this term must be universal, such as in Eq.(5) but with the prefactor determined now by the number $N_{s}$ of scalar fields with masses $m \ll T[6,13]$ :

$$
F=F_{0}-\frac{\pi^{2} N_{s}}{90 \hbar^{3}} \int d^{3} x \sqrt{-g} T^{4}-\frac{N_{s}}{144 \hbar} \int d^{3} x \sqrt{-g} T^{2}\left[\mathcal{R}+6 w^{2}\right] .
$$

In general relativity this leads to the following modification of the effective gravitational constant determined as the prefactor in front of the curvature in the free energy functional:

$$
G_{\text {eff }}^{-1}-G^{-1}(T=0)=\frac{\pi}{9 \hbar} N_{s} T^{2}
$$

\subsection{Phonon contribution in superfluid ${ }^{4}$ He.}

Phonons obey the following effective metric (which is called the acoustic metric [14]):

$$
\sqrt{-g}=\frac{1}{c^{3}} \quad, \quad g_{00}=-\left(1-\frac{\mathbf{v}_{\mathrm{s}}^{2}}{c^{2}}\right) \quad, \quad g_{i j}=\frac{1}{c^{2}} \delta_{i j} \quad, \quad g_{0 i}=-\frac{v_{\mathrm{s} i}}{c^{2}},
$$

where $c$ is the speed of sound; $\mathbf{v}_{\mathrm{s}}$ is the superfluid velocity; and we neglected the conformal factor which depends on the mass density $\rho$ of the liquid. Let 
us consider the simplest case when the velocity field is stationary. This means that there is a preferred reference frame, in which the velocity field does not depend on time, and $\mathbf{v}_{\mathrm{s}}(\mathbf{r})$ is the velocity with respect to this frame. The metric is thus stationary, but not static. Let us also assume for simplicity that $c$ and $\rho$ are space independent. Then the expression for curvature in terms of the velocity field is [15]:

$$
\mathcal{R}=\frac{1}{2}\left(\nabla \times \mathbf{v}_{\mathrm{s}}\right)^{2}+\nabla \cdot\left(\mathbf{v}_{\mathrm{s}}\left(\nabla \cdot \mathbf{v}_{\mathrm{s}}\right)\right)+\nabla \cdot\left(\left(\mathbf{v}_{\mathrm{s}} \cdot \nabla\right) \mathbf{v}_{\mathrm{s}}\right) .
$$

For pure rotation $\mathbf{v}_{\mathrm{s}}=\Omega \times \mathbf{r}$ the curvature is zero $\mathcal{R}=0$, since this corresponds to Minkowski space-time in the rotating frame. That is why let us consider the case when the velocity of the superfluid vacuum is curl-free $\left(\nabla \times \mathbf{v}_{\mathrm{s}}=0\right)$, which is the case for superfluid ${ }^{4} \mathrm{He}$ when quantized vortices are absent. Then one has

$$
\mathcal{R}=\nabla_{i} \nabla_{k}\left(v_{\mathrm{s} i} v_{\mathrm{s} k}\right)
$$

If in addition the superfluid vacuum is incompressible $\left(\nabla \cdot \mathbf{v}_{\mathrm{s}}=0\right)$, the curvature transforms to

$$
\mathcal{R}=\frac{1}{2} \Delta\left(\mathbf{v}_{\mathrm{s}}^{2}\right)=\frac{1}{2} c^{2} \Delta g_{00}=-\Delta \Phi,
$$

where $\Phi$ is the analog of the gravitational potential: $g_{00}=-\left(1+2 \Phi / c^{2}\right)$.

Let us apply Eq.(11) to the superfluid ${ }^{4} \mathrm{He}$ where $N_{s}=1$. For simplicity we consider the static effective spacetimes, i.e. those which satisfy the condition $\nabla \times\left(\mathbf{v}_{\mathrm{s}} /\left(c^{2}-v_{\mathrm{s}}^{2}\right)\right)=0$. Then integrating by parts one obtains:

$$
F-F_{0}=\int d^{3} x\left(-\frac{\pi^{2} T^{4}}{90 \hbar^{3} c^{3}}-\frac{T^{2}}{144 \hbar c^{5}} \frac{\nabla\left(\mathbf{v}_{\mathrm{s}}^{2}\right) \cdot \nabla\left(\mathbf{v}_{\mathrm{s}}^{2}\right)}{1-\frac{\mathbf{v}_{\mathrm{s}}^{2}}{c^{2}}}\right) .
$$

Let us remind that $T(\mathbf{r})=T_{0} / \sqrt{1-\mathbf{v}_{\mathrm{s}}^{2}(\mathbf{r}) / c^{2}}$ is a red-shifted temperature measured by the local observer who lives in the liquid and uses phonons for communication, while $T_{0}$ is the real temperature of the liquid, which is constant across the container in a global equilibrium.

Note that the free energy $F_{0}$ for the effective gravity itself (the hydrodynamic energy of superfluid ${ }^{4} \mathrm{He}$ in terms of $\mathbf{v}_{\mathrm{s}}$ and $\rho$ ) is very different from that in general relativity, and it certainly cannot be represented only 
in terms of the curvature term $\sqrt{-g} \mathcal{R}$. This is because the hydrodynamic equations being determined by the ultra-violet 'Planck-scale' physics are not obeying the effective acoustic metric (13). The same actually occurs in ${ }^{3} \mathrm{He}-$ A, though the effective gravity there is essentially improved as compared to that in superfluid ${ }^{4} \mathrm{He}$, and one can even identify some of the components of the Einstein action. However, in both liquids the temperature correction to the curvature term in Eqs.(17) and (10) is within the responsibility of the emerging infra-red relativistic physics and is independent of the microscopic (Planck) physics.

\subsection{Fields with different effective metrics.}

In the fermionic liquid, the velocity-dependent $T^{2} \mathcal{R}$ term (such as the second term in Eq.(17)) is also present. Actually there are even several such terms: one comes from $N_{F}=2$ chiral fermions with their metric $g_{\mu \nu}^{F}$ in Eq.(7) modified by the superfluid velocity field (see Eq.(9.13) in [4]); another one comes from the massless scalar field of the sound with its acoustic metric $g_{\mu \nu}^{s}$ in Eq.(13); and there are also two massless scalar fields of spin waves with their own 'spin-acoustic' metric. These contributions to the $T^{2}$ correction can be combined in the following way

$$
F_{2}=\frac{T_{0}^{2}}{288 \hbar} \int d^{3} x\left(\sum_{F} \frac{\sqrt{-g^{F}}}{\left|g_{00}^{F}\right|}\left(\mathcal{R}_{F}+6 w_{F}^{2}\right)-2 \sum_{s} \frac{\sqrt{-g^{s}}}{\left|g_{00}^{s}\right|}\left(\mathcal{R}_{s}+6 w_{s}^{2}\right)\right)
$$

Here $T_{0}$ is the real temperature in the liquid, which is constant in a global equilibrium, while the local red-shifted temperature is different for different quasiparticles, since it depends on the effective metric experienced by a given excitation.

\section{Discussion}

There are many condensed matter systems where the low-energy quasiparticles obey the effective Lorentzian metric. The dynamics of this effective metric is, typically, essentially different from the dynamics of the gravitational field in Einstein theory. However, the thermodynamics appears to be identical, since it is determined solely by the infra-red physics, which does not 
depend on whether the gravity is fundamental or effective. This similarity can be useful both for condensed matter and for gravity.

In particular this will be useful for the consideration of the low-temperature limit. The procedure of the gradient expansion in quantum liquids assumes that the gradients are smaller than all other quantities, in particular, the correction to the action for the metric field in Eq.(10) is valid only if the effective curvature is small enough: $\hbar^{2} \mathcal{R} \ll T^{2} \ll E_{\text {Planck }}^{2} \equiv \Delta_{0}^{2}$, i.e. when the temperture is relatively high. However, in quantum liquids or superconductors the opposite (texture-dominating) limit $T^{2} \ll \hbar^{2} \mathcal{R} \ll E_{\text {Planck }}^{2}$ is also achievable. Moreover the thermodynamic effects related to the textures (curvature) become dominating in this limit. An example is provided by the $-T^{2} \sqrt{\mathcal{B}}$ term in the free energy of the quasi-two-dimensional $d$-wave superconductor in applied magnetic field $\mathcal{B}[16]$. This term comes from the gapless fermions in the background of the $U(1)$ field, whose curvature $\mathcal{B}$ substitutes the Riemann curvature $\mathcal{R}$ of the gravitational field. It determines the thermodynamics of $d$-wave superconductors in the low- $T$ limit $T^{2} \ll \mathcal{B}$, and in particular the specific heat $C \propto T \sqrt{\mathcal{B}}$ has been measured in cuprate superconductors [17]. Whether the corresponding gravitational term $-T^{2} \sqrt{\mathcal{R}}$ appears at $T^{2} \ll \hbar^{2} \mathcal{R}$ in the free energy of quasi-two-dimensional systems with effective Lorentzian metric is the problem for future investigations.

Since these effects are determined solely by the infra-red physics, one can use for their calculations the methods developed in the relativistic theory. Since these terms are not sensitive to the dynamics of the effective gravity field, one can assume that this dynamics is governed by the Einstein equations. Then the powerful theorems derived for the Einstein gravity can be used, including the connection between the curvature terms in the free energy and the entropy of the horizon. Thus the condensed matter can serve as the arena where the connection between the gravity and thermodynamics can be exploited and developed.

We are grateful to A.A. Starobinsky for numerous discussions. This work was supported by ESF COSLAB Programme and by the Russian Foundation for Fundamental Research. AZ is grateful to the Killam Trust for its financial support. 


\section{References}

[1] A.D. Sakharov, Vacuum quantum fluctuations in curved space and the theory of gravitation, Dokl. Akad. Nauk 177, 70-71 (1967) [Sov. Phys. Dokl. 12, 1040-41 (1968)]; reprinted in Gen. Relative. Gravity 32, 365-367 (2000).

[2] T. Jacobson, Black hole entropy and induced gravity, gr-qc/9404039.

[3] V.P. Frolov, D.V. Fursaev and A.I. Zelnikov, Statistical origin of black hole entropy in induced gravity, Nucl. Phys. B 486, 339-352 (1997).

[4] G.E. Volovik, The Universe in a Helium Droplet, Clarendon Press, Oxford (2003).

[5] C. Wetterich, Gravity from spinors, hep-th/0307145.

[6] Yu.V. Gusev, A.I. Zelnikov, Finite temperature nonlocal effective action for quantum fields in curved space, Phys. Rev. D 59, 024002 (1999).

[7] D.V. Fursaev, Spectral geometry of operator polynomials and applications to QFT, hep-th/0311080; see also D.V. Fursaev, Statistical mechanics, gravity, and Euclidean theory, Nucl. Phys. B 104 (Proc. Suppl.), 33 (2002) (hep-th/0107089).

[8] J.S. Dowker and J.P. Schofield, Chemical potentials in curved space, Nucl. Phys. B 327, 267-284 (1989).

[9] V.P. Frolov and A.I. Zelnikov, Killing approximation for vacuum and thermal stress-energy tensor in static space-times, Phys. Rev. D 35, 3031-3044 (1987).

[10] J.W. York, Jr., Black-hole thermodynamics and the Euclidean Einstein action, Phys.Rev. D 33, 2092-2099 (1986).

[11] S.W. Hawking and C.J. Hunter, The gravitational Hamiltonian in the presence of non-orthogonal boundaries, Class. Quant. Grav. 13, 27352752 (1996). 
[12] M.C. Cross, A generalized Ginzburg-Landau approach to the superfluidity of ${ }^{3} \mathrm{He}$, J. Low Temp. Phys. 21, 525-534 (1975).

[13] J.S. Dowker and J.P. Schofield, High-temperature expansion of the free energy of a massive scalar field in a curved space, Phys. Rev. D 38, 3327-3329 (1988).

[14] W.G. Unruh, Experimental black-hole evaporation? Phys. Rev. Lett. 46, 1351-1354 (1981); Sonic analogue of black holes and the effects of high frequencies on black hole evaporation, Phys. Rev. D 51, 2827-2838 (1995).

[15] U.R. Fischer and M. Visser, On the space-time curvature experienced by quasiparticle excitations in the Painlevé-Gullstrand effective geometry, Ann. Phys. (N.Y.) 304, 22-39 (2003).

[16] G.E. Volovik, Fermionic entropy of the vortex state in $d$-wave superconductors, cond-mat/9702093, JETP Lett. 65, 491-496 (1997).

[17] A. Junod, B. Revaz, Y. Wang and A. Erb, Direct observation of the $d$-wave contribution to the low-temperature specific heat of the hightemperature superconductor $\mathrm{YBa}_{2} \mathrm{Cu}_{3} \mathrm{O}_{7}$, Physica B: Condensed Matter, 284-288, 1043-1044 (2000). 